\title{
XIX. Note on the cosine law of radiation
}

\section{Louis Vessot King B.A.}

To cite this article: Louis Vessot King B.A. (1912) XIX. Note on the cosine law of radiation , Philosophical Magazine Series 6, 23:134, 237-242, DOI: 10.1080/14786440208637216

To link to this article: http://dx.doi.org/10.1080/14786440208637216

册 Published online: 08 Jun 2010.

Submit your article to this journal 전

Џ Article views: 4

Q View related articles $\asymp$

4 Citing articles: 1 View citing articles 
the ground. On the other hand, many observers have found auroræ, especially rays and ray-bundles, to reach within a distance of one $\mathrm{km}$. If so, the rays must pussess an enormous penetrating power, and yet the straight-lined structure will make a radiation of the a-ray type necessary ; but it is a type not yet known from laboratory experiments.

It is a remarkable fact that the rays and ray-bundles often shoot down in between other forms of aurcra which do not reach so near to the earth. In view of our radiation hypothesis, this would mean that these very penetrating rays had about the same stiffness as ordinary $\alpha$-rays, or

$$
\frac{m v}{n e}=\frac{m_{0} v_{0}}{2 e} \text { (approximately), }
$$

where $m$ and $v$ are respectively the mass and velocity of the solar rays and ne the charge of each particle; $m_{0}, v_{0}$, and $2 e$ are the corresponding quantities for $\alpha$-rays.

From what we know about the charge carried by the small ions and by the positive rays in vacuum-tubes, $n$ ought to be a very small number. Then in order to explain the great penetrating power of the solar rays, we should have to suppose a carrier smaller than a helium atom, but moving with a velocity so much grearer than that of $\alpha$-rays that the above relation is fulfilled. The carrier might either be hydrogen or aome gas lighter than hydrogen, and the possibility suggests itself that coronium may form the carrier of the most penetrating solar rays.

XIX. Note on the Cosine Law of Radiation. By LovIs Vessot King, B.A. (Cantab.), Lecturer in Physics, McGill University *.

$\S 1$. T $\mathrm{T}$ is a well-established fact in the theory of light that the intensity in a parallel beam of homogeneous radiation travelling a distance $x$ throngb an absorbing medium is diminished according to the exponential law

$$
\mathrm{I}=\mathrm{I}_{0} e^{-\kappa x}, \text {. . . . . . . }
$$

where $I_{0}$ is the intensity over unit cross-section measured at an arbitrary origin $x=0$. $\kappa$ is a constant for the medium, called the coefficient of absorption, and depends on the wavelength of the radiation.

If the radiation emanate from a point-source $s$, we consider

* Conmunicated by Prof. H. T. Barnes, F.R.S. 
the diminution of intensity between the portions $S$ and $\mathrm{S}+d \mathrm{~S}$ of two spherical surfaces of radii $r$ and $r+d r$ cut out by a small solid angle $\omega$.

We then have

$$
\mathrm{S} d \mathrm{~T}=-\mathrm{I} d \mathrm{~S}-\mathrm{IS} \kappa d r .
$$

'The first term on the right-hand side expresses the diminution of intensity per unit cross-section due to the increase with $r$ of $\mathrm{S}$ to $\mathrm{S}+d \mathrm{~S}$, while the second term expresses the diminution of intensity by absorption in a distance $d r$.

Since $S=\omega r^{2}$, we have in a uniform medium ( $\kappa$ independent of $r$ ),

$$
\frac{d \mathrm{I}}{\mathrm{I}}=-2 \frac{d r}{r}-\kappa d r
$$

giving

$$
\mathrm{I}=\frac{\mathrm{C}}{r^{2}} e^{-\kappa r}
$$

where $(Y$ is a constant of integration.

As long as $\mathrm{S}$ remains in the same medium as the pointsource $s$, we may write the above in the form

$$
\mathrm{I}=\frac{s}{r^{2}} e^{-\kappa r}, \quad . \quad \cdot \quad . \quad \cdot .
$$

where $s$ is the intensity across unit-area at unit-distance when there is no absorption $(\kappa=0)$, in which case (2) simply expresses the law of inverse squares.

Fig. 1.

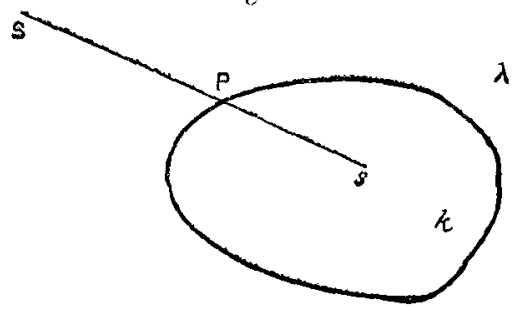

Suppose \& to be in a uniform medium whose coefficient of absorption is $\kappa$, while the intensity is measured in a medium of coefficient $\lambda$, the two media being separated by a surface cutting $s \mathrm{~S}$ at a point $\mathrm{P}$ such that $s \mathrm{P}=\mathrm{R}$. Then provided we neglect effects of refraction and reflexion at the bounding surface, a condition which is justifiable in the application to be dealt with, we may consider the path of the rays forming the pencil of small solid angle $\omega$ to be unaltered in crossing 
the boundary. Under these conditions the intensity at $\mathrm{P}$ in the medium $\kappa$ is given by

$$
\mathrm{I}_{\kappa}=\frac{s}{r^{2}} e^{-\kappa r}
$$

while the intensity in the medium $\lambda$ is given by

$$
\mathrm{I}_{\lambda}=\frac{\mathrm{C}}{r^{2}} e^{-\lambda r}
$$

The constant of integration $\mathrm{C}$ is determined by equating intensities at the boundary $r=\mathrm{R}$, which we may do if we neglect losses due to refraction and reflexion.

We find

$$
\mathrm{C}=s e^{-\kappa \mathrm{R}} e^{\lambda \mathrm{R}},
$$

so that

$$
\mathrm{I}_{\lambda}=\frac{s}{r^{2}} e^{-\kappa \mathbf{R}} e^{-\lambda(r-\mathrm{R})} . \quad . \quad . \quad . \quad . \quad .
$$

§2. The Cosine Law of Emission.-We do not have to look far for an immediate application of (3). Radiations from incandescent bodies emanate in practically all cases from a volume distribution of vibrating elements.

Fig. 2.

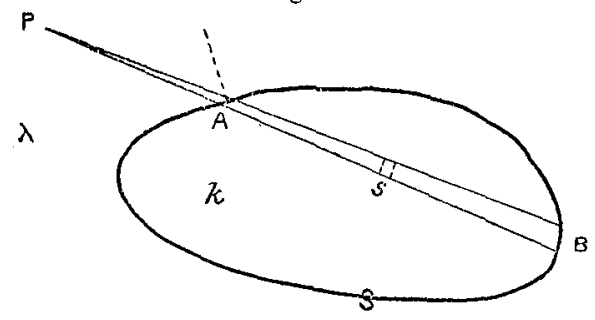

Thus, if we have an incandescent body bounded by a surface $S$, radiations from the vibrating elements in an element of volume $d v$ in the interior are practically all absorbed before reaching the surface at $A$ and thence an observer at $P$. If $\kappa$ be the coefficient of absorption of the incandescent body to its own radiations, the intensity at $P$ (situated outside $S$ in a medium of coefficient of absorption $\lambda$ ) due to a single vibrating element at $s$ is, from (3),

$$
\frac{s}{r^{2}} e^{-\kappa \mathrm{R}} \epsilon^{-\lambda(r-\mathrm{R})}
$$

where $\mathrm{R}=\mathrm{A} s$ and $r=\mathrm{P} \boldsymbol{\varepsilon}$.

If there are $\mathrm{N}$ vibrating elements per unit of volume, the 
intensity at $\mathrm{P}$ due to radiations from an element of volume $d v$ is

where $r_{1}=$ PA.

$$
d \mathrm{I}=\mathrm{N} s e^{-\lambda r_{1}} e^{-\kappa\left(r-r_{1}\right)} \frac{d v}{r^{2}}
$$

If $d v$ refer to an element of rolume cut out by the intersection of spberes of radii $r$ and $r+d r$, and centre at the vertex $P$ of a small cone of solid angle $\omega$, we have

$$
d v=r^{2} \omega d r^{2}
$$

The total intensity at $\mathrm{P}$ per unit cross-section normal to PA due to contributions from all the elements in the solid angle $\omega$ is

or

$$
\begin{aligned}
& I=N s \omega e^{-\lambda r_{1}} \int_{r_{1}}^{r_{2}} e^{-x^{\left(r-r_{1}\right)}} d r \text {, where } r_{2}=\mathrm{PB}, \\
& \mathrm{I}=\frac{\mathrm{N} s \omega}{\kappa} e^{-\lambda r_{1}}\left\{1-e^{-\kappa\left(r_{2}-r_{1}\right)}\right\} . . \quad . \quad . \quad \text { (4) }
\end{aligned}
$$

In an actual case $\lambda$ is the coefficient of absorption for air, which is very small, so that we may take $e^{-\lambda r_{1}}$ to be unity. The exponential term $e^{-\kappa\left(r_{2}-r_{1}\right)}$ represents the fraction of radiation which can penetrate the entire thickness $A B$. In solid incandescent bodies whose thickness is large compared with a wave-length this fraction is negligible.

Thus the intensity at $P$ is given very approximately by

$$
\mathrm{I}=\frac{\mathrm{N} s \omega}{\kappa}, \ldots . . .
$$

i.e. the intensity per unit cross-section of the radiation contained in a small solid angle $\omega$ viewed in any direction PA is proportional to the solid angle $\omega$. This is equivalent to stating that the whole surface $\mathrm{S}$ appears uniformly bright. Under these conditions we may replace the volume-distribution of radiating elements by a distribution of intensity over the surface $\mathrm{S}$. If $d \mathrm{~S}$ be an element of surface at $\mathrm{A}$ cut out by the cone $\omega$, and $\phi$ the angle between PA and the normal at $A$, we have

so that (5) takes the form

$$
d S=\frac{\omega r_{1}^{2}}{\cos \phi}
$$

$$
I=\frac{N s}{\kappa} \frac{d S \cos \phi}{r_{1}^{2}}
$$

i.e. if $I_{0}$ be the normal intensity at unit distance contributed 
by the element $d \mathrm{~S}$, we have the familiar form of the Cosine Law

$$
\mathrm{I}=\mathrm{I}_{0} \frac{\cos \phi}{r_{1}^{2}} . \cdot \cdot \cdot \cdot \cdot \cdot
$$

§3. In cases where the cosine law of emission fails we conclude that it is not legitimate to replace the volume distribution of vibrating elements by a surface distribution over the boundary. Wo nust, therefore, examine departures from the cosine law in the light of the more complete expression (4). We notice first of all that for incandescent bodies for which $\kappa$ is considerable, practically all the radiation which reaches $P$ comes from a very thin surface-layer, perhaps ouly a few wave-lengths in thickness. We are therefore justified in neglecting effects of reflexion and refraction at the boundary, since these can hardly obey the optical laws in so thin a transition layer.

Formula (4) show's us to some extent on what conditions the efficiency of a radiating surface depends. Practica!ly all actual means of exciting radiations in an incandescent body depend on maintaining an expenditure of energy throughout the entire volume, while the only portion of the body which contributes to the intensity of the radiation at an exterior point is an excessively thin surface-film. If it were possible to concentrate the same amount of energy throughout this thin exterior film, we shou'd expect a considerably higher efficiency. It seems probable that the high efficiency of the phosphorescent light emitted by glow-worms and fire-flies may depend on just such a surface-concentration of energy, due in this case to chemical transformations at the boundary.

It seems remarkable that the Cosine Law of Emission should not have hitherto been presented in the light of absorption theory. Fourier *, in his memoir on ' Heat,' cites a reference to one of his earlier papers, in which he probably deduced the law along lines just given. It will be noticed from (5) that the total intensity inside a cavity in an infinite solid, or at any rate in one bounded by an exterior surface so large that contributions from portions near the exterior are small compared with those from portions near the cavity, is given by

$$
\mathrm{I}=4 \pi \frac{\mathrm{N} s}{\kappa} .
$$

$I$ is independent of the shape and size of the cavity, and

* Fourier, 'Chaleur,' Paris, 1822, p. 31, Chap. 1. 'The paper referred to is in Mém. Acarl. des sc. tome v, Paris, 1826, pp. 179-213. 
also of the position of $\mathrm{P}$ in its interior. This result is employed by Fourier as a verification of the cosine law *

$\S 4$. In deducing the formula just given, we have employed the simple exponential law of absorption

$$
\mathrm{I}=\mathrm{I}_{0} e^{-\kappa r \cdot} \text {. }
$$

This by itself involves a violation of the principle of conservation of energy. In the case of gases, we know that absorption of light is accompanied by lateral scattering: according to the well-known Rayleigh law. In the case of incandescent solids the problem is very much more complicated. Each of the radiating elements, besides being subject to a primary disturbing cause, is also subject to the aggregate radiation from all the other elements in the surface S. This constitutes what Schustert has called "Radiation in a Foggy Atmosphere." The complete expression of this problem can be given in terms of an integral equation in three dimensions. This, together with some applications to the intensity of skylight, the writer hopes to communicate in a future paper.

XX. Absorption Problems in Radioactivity. By LouIs VEssoT KING, B.A. (Cantab.), Lecturer in Physics, McGill University $\ddagger$.

[Plate VII.]

$\S 1.7 \mathrm{PHE}$ intensity of $\beta$ - and $\gamma$-rays in their passage through matter decreases approximately according to the exponential law

$$
\mathrm{I}=\mathrm{I}_{0} e^{-\kappa x}, \text {. . . . . }
$$

$I_{0}$ being the intensity at an arbitrary origin $x=0$ as measured by the ionizing power of the rays, and $\kappa$ the coefficient of absorption. Under these conditions it can easily be shown that the intensity at a distance $r$ from a point-source of rays in a medium whose coefficient of absorption is $\kappa$ is given by

$$
\mathrm{I}=\frac{s}{r^{2}} e^{-\kappa r}, \quad \cdot \quad \cdot \quad \cdot \quad \cdot
$$

$s$ being the intensity at anit distance when there is no absorption.

* Fourier, loc. cit.p. $31, \S 47$.

† Schuster, Astrophysical Journal, Jan. 1905, p. 1.

‡ Communicated by Prof. H. T. Barnes, F.R.S. 